\title{
Nuclear Fusion of Protons with Ions of Boron*
}

\author{
Alessandro G. Ruggiero \\ Brookhaven National Laboratory \\ Upton, Long Island, New York 1 1973, USA
}

\section{Abstract}

This paper describes a method of extracting energy from the fusion events occurring between the collision of a beam of protons with a beam of ions of Boron 11. The two beams are circulating in separated and intersecting storage rings where they collide head-on in a common long-straight section. Requirements on the luminosity of the low-energy collider are shown. Limitations due to space-charge and intrabeam scattering are described. Very fast electron cooling is needed to compensate these effects.

\section{INTRODUCTION}

One of the most interesting fusion reaction [1] is a proton colliding with an ion of Boron 11. For a very brief period of time, an ion of Carbon 12 is formed which then immediately decays in three $\alpha$ particles. In order for this reaction to occur, assuming the ion of Boron at rest, the proton needs a sufficiently large energy. There is a broad resonance [2] centered around $675 \mathrm{keV}$ which exhibits a large cross-section of 0.9 barn. The low energy combined with the large fusion crosssection makes the reaction a good choice as a method for obtaining fusion nuclear power. Once the lowest bound state is reached, a total energy of $8.7 \mathrm{MeV}$ is released under the form of kinetic energy given to the $\alpha$ particles.

Since it is relatively easy to control the energy of the particle beams with today's accelerator technology, the fusion reaction here proposed can be easily ignited with no other possible channels of interaction involved. In particular no neutrons or gamma rays are produced. Another interesting feature is the large state of electric charge $(\mathrm{Z}=6)$ of the final products which suggests methods employing electricity for the immediate conversion of the nuclear energy to electric power.

A method [3] which makes use of two beams, one of protons and one of ions of Boron, colliding periodically with each other is presented in Section II. We report on the beam requirements for a small size device producing a $1 \mathrm{MW}$ power in Section III. Section IV is a discussion of the limitations due to space-charge and of the possible cure by beam-charge neutralization with electrons. The effects of intrabean scattering are discussed in Section V. Finally, electron cooling requirements are reported in Section VI.

\section{THE COLLIDER}

This is shown in Figure 1. The proton beam is generated with hydrogen-ion sources followed by RFQ devices [4] operating at few tens to few hundred of $\mathrm{MHz}$. Average currents of a hundred milliampere are possible. The proton beam is injected in a cooling and storage ring. Cooling is needed to reduce or to maintain the beam transverse dimensions to sufficiently small values to enhance the collision events in the collider.

* Work performed under the auspices of U.S. DOE.
The beam of ions $B^{11}$ is also prepared already completely stripped from similar RFQ devices fed by ECR sources. The ECR sources [5] should be capable to deliver an amount of beam intensity matching the one of the proton beam. Also the ion beam will be injected in its own cooling and storage ring which is placed sidewise next to the proton ring. There is thus a continuous streaming of beams at both sides, from the sources to the RFQ's, to the storage rings and to the collision region. The two beams are circulating in the same direction in the respective storage rings and collide head-on in the common straight section of the collider. In this location protons and ions will fuse. During collision the two beams have essentially the same bunching structure, transverse dimensions and intensity. The long straight section where the two beams collide is surrounded by the reactor vessel itself.

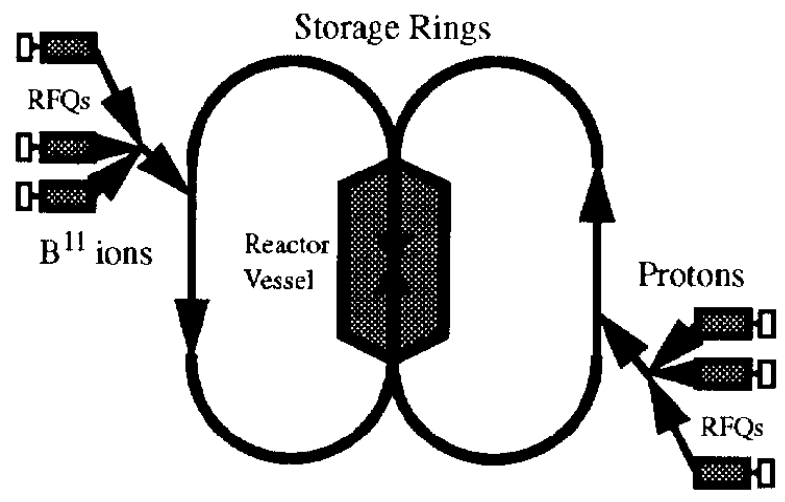

Figure 1. Nuclear Reactor with Colliding Beams

Each storage ring has a circumference $2 \pi \mathrm{R}=3.3 \mathrm{~m}$ and is made of a sequence of 16 FODO cells, each having a phase advance of $90^{\circ}$ and a length of $21 \mathrm{~cm}$. Quadrupoles and dipoles are made of permanent magnets. Quadrupoles are $2 \mathrm{~cm}$ long. There are 16 dipoles, each $8 \mathrm{~cm}$ long, placed in the arcs. The long straights are made of 4 cells each without dipoles. Matching is assumed to provide zero dispersion in the long straights.

\section{BEAM REQUIREMENTS}

Let $T_{p}$ and $T_{B}$ be the energies respectively of protons and Boron ions. We require $T_{p}+T_{B}=675 \mathrm{keV}$. The solution yielding equal velocities is $T_{p}=56 \mathrm{keV}$ and $T_{B}=619 \mathrm{keV}$, corresponding to the velocity $\beta=0.011$. Let $N$ be the number of particles in each beam that at any one time travel in the interaction region. Let also $S$ be the common cross-section of the two beams where they collide. The frequency of particle encounter $f_{e n c}$ is the revolution frequency. The number of fusion events per unit of time in this configuration is then given by

$$
\mathrm{dn} / \mathrm{dt}=\sigma\left(\mathrm{N}^{2} / \mathrm{S}\right) \mathrm{f}_{\mathrm{enc}}=\sigma \mathrm{L}
$$

where $\sigma=0.9 \times 10^{-24} \mathrm{~cm}^{2}$ is the fusion cross-section and $\mathrm{L}$ is 
the luminosity of the collision.

If each fusion event releases an amount of energy $\mathrm{W}$, the instantaneous power produced is

$$
P_{g}=W(d n / d t)
$$

At the same time there is a depletion of both beams which will occur at the rate

$$
\mathrm{dN} / \mathrm{dt}=-\mathrm{dn} / \mathrm{dt}
$$

The ion sources have then to replenish the beams also at these rates. One can estimate the amount of power, respectively $P_{p}$ and $\mathrm{P}_{\mathrm{B}}$, which will be required just for the beam production

$$
\mathrm{P}_{\mathrm{p}}=\mathrm{T}_{\mathrm{p}}(\mathrm{dn} / \mathrm{dt}) \text { and } \mathrm{P}_{\mathrm{B}}=\mathrm{T}_{\mathrm{B}}(\mathrm{dn} / \mathrm{dt})
$$

To this we add the power $P_{\text {acc }}$ that is dissipated for the operation of the entire complex. The equation for the power $P_{\text {exc }}$ produced in excess is

$$
\begin{aligned}
P_{\text {exc }} & =P_{g}-P_{p}-P_{B}-P_{\text {acc }} \\
& =\left(W-T_{p}-T_{B}\right)(d n / d t)-P_{a c c}
\end{aligned}
$$

It is seen that, in order for the process to produce useful power, $\mathrm{P}_{\mathrm{exc}}$ has to be positive, which requires that

$$
\mathrm{T}_{\mathrm{p}}+\mathrm{T}_{\mathrm{B}}<\mathrm{W}
$$

In our case $\mathrm{W}=8.7 \mathrm{MeV}$ and $\mathrm{T}_{\mathrm{p}}+\mathrm{T}_{\mathrm{B}}=675 \mathrm{keV}$. Also

$$
\sigma \mathrm{L}>\mathrm{P}_{\mathrm{acc}} /\left(\mathrm{W}-\mathrm{T}_{\mathrm{p}}-\mathrm{T}_{\mathrm{B}}\right)
$$

Neglecting the amounts of beam power, a break-even situation is obtained when the power delivered equals the power just needed for the operation of the system, which gives

$$
\mathrm{L}=\mathrm{P}_{\mathrm{acc}} /(\sigma \mathrm{W})
$$

Since it is not clear what value one should take for the operation power $P_{\text {acc }}$ we shall assume that the nuclear power from the collisions of the two beams is entirely available as excess power. We shall set as a goal $P_{\mathrm{exc}}=1 \mathrm{MW}$, so that the required luminosity of the collision is

$$
\mathrm{L}=\mathrm{P}_{\mathrm{exc}} /(\sigma \mathrm{W}) \sim 10^{42} \mathrm{~cm}^{-2} \mathrm{~s}^{-1}
$$

At the same time, the rate of fusion events is

$$
\mathrm{dn} / \mathrm{dt} \sim 10^{18} \text { events per second }
$$

This is also equal to the rate of depletion of the beams and to the rate particles are produced at their sources and are entering the system. Beam currents at injection are not excessive since they are about $100 \mathrm{~mA}$-particle for both types of beam. Protons can be produced with a single source; the production of ions of boron requires improvement of the sources available. Eventually several ECR sources can be used in parallel.

If the circumference $2 \pi \mathrm{R}$ of the collider is 3.3 meter, the frequency of encounter $\mathrm{f}_{\text {enc }}$ is $1 \mathrm{MHz}$. Assuming a beam crosssection at collision $S=10^{-4} \mathrm{~cm}^{2}$, then each beam is made of about $\mathrm{N}=10^{16}$ particles. Each particle will spend in average ten milliseconds in the collider, that is it will survive in average ten thousand revolutions.

\section{SPACE CHARGE LIMITATIONS}

There is a very serious limitation due to the beam spacecharge. It is customary to measure this limit [6] in terms of the maximum value $\Delta v$ that can be allowed for the betatron-tune depression

$$
\Delta v=N r_{p} Q^{2} /\left(2 \beta^{2} \gamma^{3} B A \varepsilon\right)
$$

where $r_{p}=1.535 \times 10^{-18} \mathrm{~m}$ is the classical radius of a proton, $\mathrm{N}$ the total number of particles, $Q$ the charge state and $A$ the mass number of the particle specie, $B$ the bunching factor defined as the ratio of average beam current to the peak current and which we take here to be unit, and $\varepsilon$ is the full betatron emittance. A reasonable limit of $\Delta v$ is about a unit. To keep the beam within the space-charge limit, one derives $\varepsilon>50 \pi \mathrm{m}$ rad.

It is very difficult to achieve the required beam spot size of $0.1 \mathrm{~mm}$ at the interaction region with such large beam emittance. An ordinarily conceived method is the focussing of the particle motion with quadrupole magnets placed on both sides. Typically a small value of $\beta^{*}$ is few centimeters. In turn this requires a beam emittance $\varepsilon=10^{-6} \pi \mathrm{m}$ rad, that is eight orders of magnitude smaller than the space-charge limit. This figure is also about what one can expect for the beam emittance from the source and therefore at injection into the storage rings.

It is possible to compensate space-charge effects with neutralization of the beam electric charge by trapping electrons produced by ionization of the atoms of the residual gas in the vacuum chamber of the storage rings. It has been experimentally demonstrated [7] at INP (Novosibirsk, Russia) that it is possible to store with this method a current ten times larger than the value otherwise set by the space-charge limit. A factor of a thousand is also believed possible.

Let $P_{m m H g}$ be the vacuum in the storage rings expressed in $\mathrm{mmHg}$. At room temperature, the number of molecules per unit of volume is given by

$$
n_{\mathrm{mol}}=\left(3 \times 10^{16} / \mathrm{cm}^{3}\right) \mathrm{P}_{\mathrm{mmHg}}
$$

which corresponds to the following density

$$
\rho=\left(1.4 \times 10^{-6} \mathrm{~g} / \mathrm{cm}^{3}\right) \mathrm{P}_{\mathrm{mmHg}} \text {. }
$$

A vacuum pressure of $1 \times 10^{-8} \mathrm{mmHg}$ corresponds to an energy loss of less than $1 \mathrm{keV}$, by atom excitation and ionization, for a particle spending the average 10 milliseconds in the storage ring. This vacuum pressure is thus adequate and it is assumed in the following.

The ionization rate can be estimated with the equation

$$
\mathrm{dn}_{\mathrm{i}} / \mathrm{dt}=\beta \mathrm{c} \sigma_{\mathbf{l}} \mathbf{n}_{\text {mol }} \mathrm{N}
$$

where $\sigma_{1}=2.5 \times 10^{-18} \mathrm{~cm}^{2}$ is the ionization cross-section. With the vacuum pressure of $10^{-8} \mathrm{mmHg}$, the number of electrons produced is $10^{15}$ per second. It takes about ten seconds to produce a number of electrons equal to number of particles to achieve complete space-charge neutralization. As the electrons are being produced and accumulate within the beam dimensions, the potential barrier decreases continuously until vanishes. Beyond that point the electrons produced in excess will leave the beam and the neutralization should persist for a long 
period of time. There should be an initial period where the beam is slowly established toward full intensity and density in combination of electron cooling and ionization. It is important that the ionization method for space-charge neutralization is also used in a controllable fashion during cooling to avoid that the space-charge limit is exceeded during the storage.

\section{INTRABEAM SCATTERING}

Because of the large density, particles in the same beam will scatter with each other by Coulomb repulsion. This effect will cause a considerable increase of the beam dimensions and particle losses as well.

We have estimated the rates of diffusion using available computer programs for the purpose [8]. The storage rings operate below the transition energy and the following features have been observed. For sufficiently small energy spread $\left(<10^{-4}\right)$ there is actually a reduction of the transverse dimensions at cost of increasing the energy spread. The situation is inverted for larger initial energy spreads $\left(>10^{-3}\right.$ ) when the beam emittance will grow and the momentum spread actually reduces. There is an intermediate range where growth occurs in all dimensions. The exact behavior depends of course on the choice of the storage ring lattice. For large energy spreads, the relative growth rate of the betatron emittance is about $10^{9} \mathrm{~s}^{-1}$.

There are several methods to cope with this large diffusion caused by intrabeam scattering. We mention below few of the possibilities.

A. Electron Cooling. This will be discussed in the next Section VI.

$B$. Chose the storage ring lattice and the beam parameters in such a way that the intermediate energy-spread range discussed above vanishes, and an equilibrium is found where the diffusion rates are identically zero in all dimensions.

C. Diffusion rates are directly proportional to the Coulomb Log, which is the logarithm of the ratio of the largest to the lowest impact parameters

$$
\log =\log \left(b_{\max } / b_{\min }\right) \text {. }
$$

It may be possible to chose beam dimensions so that the two impact parameters are equal, in which case $\log =0$.

The smallest impact parameter is given by the largest scattering angle

$$
b_{\min }=2 \pi Q^{2} r_{0} \beta_{L} / A \varepsilon \beta^{2}
$$

where $\beta_{\mathrm{L}}$ is the average value of the amplitude lattice function. For a very dense beam, the large impact parameter is given by the Debye radius, that is the range above which particles are effectively screened from each other

$$
\mathrm{b}_{\max }=\varepsilon \beta \sqrt{\mathrm{AR} / 2 \pi \mathrm{Q}^{2} \mathrm{r}_{0} \mathrm{~N}}
$$

It is seen that if $\varepsilon=0.1 \pi \mathrm{mm}$ mrad and all the other parameters are unchanged, then $b_{\max }=b_{\text {min }}$.

D. It may be possible [9] to employ very fast cooling to "freeze" the particles in the beam in a cold, high-ordered state, where they are essentially equally spaced ("crystalline beam").
Once this configuration is reached, all particles would be effectively screened from each other, and scattering among each other is thus removed. Of course it remains to check what happens to the ordered state when the two beams collide with each other.

\section{ELECTRON COOLING}

The most important requirement, in order for cooling to be effective, is to provide velocity matching between the ion beam and the electrons. Since $\beta=0.011$ this corresponds to an electron kinetic energy of only $30 \mathrm{eV}$. Whether a beam with such low energy is feasible over an extended length depends on other parameters like intensity and transverse dimensions which in turn depend on the required cooling rate. Cooling should proceed to maintain the beam at the space charge limit. Moreover cooling has to balance the diffusion rates due to intrabeam scattering which happens to be by far the most important effect. Thus a cooling rate $\lambda=10^{9} \mathrm{~s}^{-1}$ may be required.

The formula for the cooling rate $[10]$ is

$$
\lambda=\left(4 \pi Q^{2} r_{c} r_{p} L \mu J / e\right) /\left(A \beta^{4} \gamma^{5} \theta^{3}\right)
$$

where $r_{c}=2.818 \times 10^{-15} \mathrm{~m}$ is the electron classical radius, $\mu$ the ratio of the length of the electron beam to the circumference, $J$ the electron beam density, $\mathrm{L} \sim 10$ the Coulomb Log, and $\theta$ the angular divergence of the ion beam which we can assume to be larger than the angular divergence of the electron beam. With our parameters $\theta=1 \mathrm{mrad}$. If we take $\mu=10 \%$, the electron beam density for the cooling of the protons is $J=4 \mathrm{~A} / \mathrm{cm}^{2}$. With a radius of 1 millimeter, the electron current is then $0.13 \mathrm{~A}$.

\section{ACKNOWLEDGMENTS}

The author of this paper wishes to tank Dr. Antonio Dainelli of the Laboratori Nazionali di Legnaro (INFN), Padua, Italy, for very valuable and informative discussions.

\section{REFERENCES}

[1] L.M. Lidsky, "The Trouble with Fusion", Technology Review, Oct 1983. Edited by M.I.T.

[2] F. Ajzenberg-Selove and C. Langell Busch, Nuclear Physics $\mathrm{A336}$, p. 73 (1980).

[3] A.G. Ruggiero, "Nuclear Fusion of Protons with Boron", BNL - 47989 and AD/AP-48 (Sept. 1992).

[4] J.W. Staples, "RFQs - An Introduction", AIP Conf. Proc. 249, Vol.2, p. 1483. The Physics of Particle Accelerators. (1992).

[5] R. Geller, "ECR Source Scaling Concepts", IEEE Particle Accel. Conf. March 1989, Chicago, IL. Vol. 2, p. 1088.

[6] L.J. Laslett, "On Intensity Limitations imposed by Transverse Space Charge Effects in Circular Particle Accelerators", BNL 7534 (1963).

[7] G.I. Dimov and V.E. Chupriyanov, Particle Accelerators, 14 , p. 155 (1984).

[8] M. Martini, PS/AA/Note 84-7. CERN, May 1984.

[9] A.G. Ruggiero, these proceedings.

[10] T. Ogino and A.G. Ruggiero, Particle Accelerators, 10, p. $197(1980)$. 